\title{
Sleep as a Window into the World of Fibromyalgia Syndrome
}

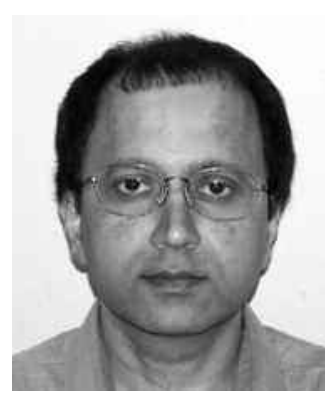

One of the most clinically problematic challenges in the management of fibromyalgia syndrome (FM) is the unrefreshing nature of sleep. The problem goes beyond misperceiving the duration or quality of sleep, to a point where patients may feel worse after sleep - a complete loss of the restorative power of sleep. This clinical feature has been associated with changes in sleep that are not readily determined by conventional sleep stages and scoring, but may be identified by a number of complementary analyses. In essence, they all capture in some form the dominance of low amplitude fluctuations in physiological signals during sleep ${ }^{1,2}$. "Low" is a relative term - pathological sleep, a good example of which is sleep apnea, is dominated by oscillations recurring in nearly every signal trace, every 25-35 seconds. There is a separate set of oscillations at a higher frequency that dominates slow-wave sleep and unfragmented N2 non-rapid eye movement (NREM) sleep, centered on respiration, best seen as strong sinus arrhythmia and breath-to-breath respiratory modulation of blood pressure during periods of stable breathing ${ }^{3}$.

In the electroencephalographic (EEG) domain, the low frequency oscillations are detected by scoring the cyclic alternating pattern, or $\mathrm{CAP}^{4}$. These periods are dominated by phasic EEG complexes. A1 CAP is made up of slower wave forms, A3 fast/arousing waveforms, and A2 admixtures. An increase in A2/A3 CAP is seen in a range of sleep-fragmenting conditions, including FM, sleep apnea, epilepsy, and auditory stimulation ${ }^{4}$. CAP is reduced during recovery sleep following sleep deprivation ${ }^{5}$, during the rebound of deeper sleep during positive airway pressure titration for sleep apnea ${ }^{6}$, and following use of sedatives ${ }^{7}$. Non-CAP are periods of NREM sleep with a paucity of phasic EEG activity. Such periods are associated with stable breathing and blood pressure dipping ${ }^{8}$. Heart rate variability analysis shows that CAP is associated with sympathetic dominance, and non-CAP with parasympathetic dominance $^{9,10}$. Fragmented REM sleep takes on a CAP-like pattern in terms of oscillatory pattern dominance, but
CAP/non-CAP are primarily NREM sleep metrics. An electrocardiogram (ECG)-based technique of mapping coupled oscillations during sleep correlates with CAP architecture, such that high frequency coupling is associated with non-CAP, and low frequency coupling with $\mathrm{CAP}^{3}$. The former is reduced in patients with $\mathrm{FM}^{10}$ and depression ${ }^{11}$, and is associated positively with glucose disposition index, suggesting that these types of EEG and autonomic metrics tell us something important about fundamental sleep processes.

The CAP rate (the percentage of NREM sleep in CAP) is not going to be a specific diagnostic metric. Can CAP be used as a biomarker for treatment effects in specific conditions like depression, insomnia, sleep apnea, or FM? If there is an elevated CAP rate (normalized or otherwise) and a treatment does not reduce it, improvements in sleep quality have not likely occurred. CAP and low frequency coupling (which overlap, but not exactly) may be a final common pathway of fragmented and nonrestorative or "ineffective" sleep - sleep unable to perform its many functions.

The article by Moldofsky's group in this issue of The Journal suggests that CAP may be a useful biomarker for tracking treatment effects in $\mathrm{FM}^{12}$. Using cyclobenzaprine 1-4 mg, A2/A3 CAP normalized to total CAP appeared to be a useful biomarker that predicted positive treatment effects. Scoring CAP manually by visual inspection of polysomnograms is an expensive exercise and a labor of love, impractical for large datasets. Some automated software such as within RemLogic (Natus/Embla Systems, Inc., Denver, CO, USA) is commercially available although not extensively used and validated. The ECG-spectrogram is automated and could serve a similar function, but I suspect the measures could be complementary.

What could CAP and related biomarkers tell us about the pathology of FM and pain processing? The cortical pathways modulating autonomic activity and pain processing overlap to a great extent, and include the insula, the anterior cingulate cortex, and the ventromedial prefrontal cortex ${ }^{13,14,15}$. The presence of excessive phasic EEG activity

See Effects of cyclobenzaprine in FM, page 2653 
may reflect enhanced or abnormal activity within this network visualized through the window of sleep. Thus, the unique signal/noise characteristics of sleep, including the propensity to group oscillations within certain limited frequency ranges, may provide a window into FM and similar syndromes. It is possible that CAP and biological processes associated with it are amplifier systems, such that CAP sleep sustains in some way abnormal nociceptive processing. Thus, sleep may be both an innocent bystander and a perpetrator in patients with FM. A case can be made that any treatment that does not reduce CAP A2/A3 may not improve symptoms of FM, regardless of where the site of action is. As CAP sleep is also associated with non-dipping of blood pressure $^{8}$, one wonders if patients with FM could be at greater risk of poor outcomes, such as vascular and cognitive, associated with non-dipping in general.

Simply suppressing or altering CAP patterns may not be enough. For example, the benzodiazepines and non-benzodiazepine GABA receptor modulators reduce $\mathrm{CAP}^{12}$, but do not seem to have unique clinical utility in FM. The effects of sodium oxybate ${ }^{16}$ on EEG CAP would be interesting to know, although I predict there would be a reduction in A2/A3 CAP.

The best research inevitably raises more questions than it answers. Using sleep as a medium to view pathophysiological processes in FM, and using sleep-state biomarkers to enhance management, seems to have new potential to be a rewarding strategy.

ROBERT JOSEPH THOMAS, MD, Assistant Professor of Medicine, Beth Israel Deaconess Medical Center and Harvard Medical School, 330 Brookline Avenue,

Boston, Massachusetts 02215, USA

Address correspondence to Dr. Thomas.

E-mail:rthomas1@bidmc.harvard.edu

\section{REFERENCES}

1. Thomas RJ, Mietus JE, Peng CK, Goldberger AL, Crofford LJ, Chervin RD. Impaired sleep quality in fibromyalgia: Detection and quantification with ECG-based cardiopulmonary coupling spectrograms. Sleep Med 2010;11:497-8.

2. Rizzi M, Sarzi-Puttini P, Atzeni F, Capsoni F, Andreoli A, Pecis M, et al. Cyclic alternating pattern: A new marker of sleep alteration in patients with fibromyalgia? J Rheumatol 2004;31:1193-9.
3. Thomas RJ, Mietus JE, Peng CK, Goldberger AL. An electrocardiogram-based technique to assess cardiopulmonary coupling during sleep. Sleep 2005;28:1151-61.

4. Parrino L, Ferri R, Bruni O, Terzano MG. Cyclic alternating pattern (CAP): The marker of sleep instability. Sleep Med Rev 2011 May 24. [Epub ahead of print]

5. De Gennaro L, Ferrara M, Spadini V, Curcio G, Cristiani R, Bertini $\mathrm{M}$. The cyclic alternating pattern decreases as a consequence of total sleep deprivation and correlates with EEG arousals. Neuropsychobiology 2002;45:95-8.

6. Parrino L, Smerieri A, Boselli M, Spaggiari MC, Terzano MG. Sleep reactivity during acute nasal CPAP in obstructive sleep apnea syndrome. Neurology 2000;54:1633-40.

7. Terzano MG, Parrino L. Effect of hypnotic drugs on sleep architecture. Pol J Pharmacol 1994;46:487-90.

8. Iellamo F, Placidi F, Marciani MG, Romigi A, Tombini M, Aquilani $\mathrm{S}$, et al. Baroreflex buffering of sympathetic activation during sleep: evidence from autonomic assessment of sleep macroarchitecture and microarchitecture. Hypertension 2004;43:814-9.

9. Ferini-Strambi L, Bianchi A, Zucconi M, Oldani A, Castronovo V, Smirne S. The impact of cyclic alternating pattern on heart rate variability during sleep in healthy young adults. Clin Neurophysiol 2000;111:99-101

10. Ferri R, Parrino L, Smerieri A, Terzano MG, Elia M, Musumeci SA, et al. Cyclic alternating pattern and spectral analysis of heart rate variability during normal sleep. J Sleep Res 2000;9:13-8.

11. Yang AC, Yang CH, Hong CJ, Tsai SJ, Kuo CH, Peng CK, et al. Sleep state instabilities in major depressive disorder: Detection and quantification with electrocardiogram-based cardiopulmonary coupling analysis. Psychophysiology 2010 Jul 6. [Epub ahead of print]

12. Moldofsky H, Harris HW, Archambault WT, Kwong T, Lederman S. Effects of bedtime very low dose cyclobenzaprine on symptoms and sleep physiology in patients with fibromyalgia syndrome: A double-blind randomized placebo-controlled study. J Rheumatol 2011;39:2653-63.

13. Critchley HD. Neural mechanisms of autonomic, affective, and cognitive integration. J Comp Neurol 2005;493:154-66.

14. Critchley HD, Nagai Y, Gray MA, Mathias CJ. Dissecting axes of autonomic control in humans: Insights from neuroimaging. Auton Neurosci 2011;161:34-42.

15. Maihofner C, Seifert F, Decol R. Activation of central sympathetic networks during innocuous and noxious somatosensory stimulation. Neuroimage 2011;55:216-24.

16. Russell IJ, Holman AJ, Swick TJ, Alvarez-Horine S, Wang YG, Guinta D. Sodium oxybate reduces pain, fatigue, and sleep disturbance and improves functionality in fibromyalgia: Results from a 14-week, randomized, double-blind, placebo-controlled study. Pain 2011;152:1007-17.

J Rheumatol 2011;38:2499-500; doi:3899/jrheum.111314 